\title{
Full Abstract to Published Manuscript: Evaluation of the Scientific Impact of the American Society of Breast Surgeons Conference
}

\author{
Zachary J. Brown, DO, Amy Li, Chengli Shen, MD, PhD, and Ko Un Park, MD $\mathbb{0}$ \\ Division of Surgical Oncology, Department of Surgery, The Ohio State University Wexner Medical Center, Columbus, OH
}

\begin{abstract}
Background. The scientific rigor of the abstracts presented at the American Society of Breast Surgeons (ASBrS) annual meeting has not been recently evaluated. In this study, we sought to determine the rate at which abstracts presented at the 2017 and 2018 ASBrS meetings were published in peer-reviewed journals, and compared the rates with breast abstracts presented at the 2018 Society of Surgical Oncology (SSO) meeting.

Methods. Abstracts from the 2017 and 2018 ASBrS and 2018 SSO conferences were searched in PubMed for published manuscripts using the abstract title and/or first or last author.

Results. In 2017, $21.6 \%$ of the 268 abstracts presented at the ASBrS conference resulted in full publication, compared with $36.6 \%$ of the 273 abstracts presented at the 2018 ASBrS conference, resulting in a significant difference in the publication rate $(p<0.001)$. Of the 158 abstracts published from the 2017 and 2018 meetings, 75 (47.8\%) were published in Annals of Surgical Oncology (ASO). There was no correlation between impact factor and time to publication. Oral presentations and quick shots were more
\end{abstract}

Abstract presented at the 2021 American Society of Breast Surgeons Conference.

Supplementary Information The online version contains supplementary material available at https://doi.org/10.1245/s10434021-10493-1.

(C) Society of Surgical Oncology 2021

First Received: 31 March 2021

Accepted: 1 July 2021;

Published Online: 21 July 2021

K. U. Park, MD

e-mail: koun.park@osumc.edu; park.2835@osu.edu likely to be published than poster presentations, and oral presentations were more likely to be published in higherimpact journals. The 2018 SSO meetings resulted in 54 of $111(48.6 \%)$ breast abstracts leading to full publication.

Conclusion. Approximately $29.2 \%$ of the abstracts presented at the ASBrS 2017 and 2018 conferences resulted in a published manuscript. A higher publication rate in higher impact journals for oral presentations indicates that the abstract review process properly stratifies the research.

For many years, medical conferences have been a mainstay of medical education and a means of dissemination of advances in research, education, training, and policy. Prior to the coronavirus disease 2019 (COVID-19) pandemic, there were a plethora of medical conferences worldwide, including international, national, and local meetings. At these conferences, abstracts are presented to share new medical information and the peer-review process may vary in the scientific rigor under which the abstracts are reviewed. ${ }^{1,2}$ There has been speculation that some abstracts may be accepted based on mere merit of the authors or prestige of the institution from which they originate. ${ }^{3}$ Additionally, many abstracts have been found to have significant changes, with increased quality, when they undergo a more extensive peer-review process and are published as full manuscripts. ${ }^{1,4-6}$ As such, many authors argue that unpublished data that have not undergone the full peer-review process should not be included in metaanalyses as this could obstruct the final results. ${ }^{7}$

A recent Cochrane review found that only $37.3 \%$ of abstracts were published as a full manuscript. ${ }^{8}$ The scientific rigor of the abstracts presented at the American Society of Breast Surgeons (ASBrS) annual meeting has not been recently evaluated. In this study, we sought to determine the rate at which abstracts presented at the 2017 
and 2018 ASBrS meetings were published in a peer-reviewed journal. In addition, the publication rate of abstracts published at the ASBrS meetings were compared with abstracts on breast topics presented at the Society of Surgical Oncology (SSO) Symposium.

\section{METHODS}

The ASBrS and SSO host annual scientific conferences in the US with scientific content from research abstracts that are peer reviewed (evaluated by individuals working in the same field for quality and scientific rigor). The abstracts are divided into oral presentations, quick shot presentations, and poster presentations. Select abstracts are published in the host journal, Annals of Surgical Oncology (ASO).

Abstracts presented at the 2017 and 2018 ASBrS conferences, as well as abstracts involving the topic of breast at the 2018 SSO annual conference, as outlined in the SSO Conference Abstract Book, which lists abstracts by disease site, were included in our analysis. Abstracts were obtained through published ASBrS and SSO programs from the respective years. Video presentations were omitted from the analysis. A comprehensive search of the abstracts was conducted electronically through PubMed using the title, keywords, and/or first or last author. Publications needed to match abstracts in terms of similarity in corresponding title, author, hypothesis, and/or results, and be available online by the search date of December 2020. The publication date was determined by the online publication date indicated on PubMed. Journals included were those that are peer-reviewed and available for searching on PubMed, regardless of open access status. Journal Citation Reports (JCR) from Web of Science was used to provide the 2019 impact factors of affiliated journals. The lag time to publication was defined as the time interval between date of the conference to online publication date. International studies were defined as taking place outside of the US, while multinational studies were performed in institutions from different countries.

Continuous variables were presented as the mean $\pm \mathrm{s}$ tandard deviation, and one-way analysis of variance (ANOVA) with Tukey's multiple comparisons test was used for the comparison of continuous variables. Categorical variables were expressed as frequencies and percentages, and Fisher's exact test was employed for comparisons between the two meetings. Statistical significance was set at two-sided $\alpha<0.05$. All statistical analyses were performed using STATA version 16 (StataCorp LLC, College Station, TX, USA).

\section{RESULTS}

Overall, 541 abstracts were presented at the 2017 and 2018 ASBrS conferences, of which 158 (29.2\%) were published as a full manuscript in a peer-reviewed journal. In 2017, 268 abstracts were presented at the ASBrS conference, of which $58(21.6 \%)$ resulted in a full publication. In comparison, 273 abstracts were presented at the ASBrS conference in 2018 and $100(36.6 \%)$ resulted in a full publication, resulting in a significant increase in publication rate between the 2017 and 2018 ASBrS meetings $(p<0.001)$.

At the 2017 and 2018 ASBrS conferences, there were 20 oral presentations in total, of which $13(65 \%)$ resulted in full publication, 18 quick shots, of which $13(72 \%)$ resulted in full publication, and 503 posters, with 132 (26\%) resulting in full publication. A total of 158 abstracts resulted in full publication, $132(83.5 \%)$ of which were presented as posters, $13(8.2 \%)$ as oral presentations, and $13(8.2 \%)$ as quick shot presentations. Oral presentations and quick shot presentations were more likely to be published in any journal than poster presentations. There was no association with the type of data (clinical trial, retrospective date, or basic science) or institution type leading to publication in any journal (Table 1).

We combined the 2017 and 2018 ASBrS abstracts that were published as manuscripts to determine the studies that were published in ASO, the official journal of the SSO and ASBrS. Ultimately, 75 (47.47\%) abstracts that were

TABLE 1 Factors associated with publication of American Society of Breast Surgeons Conference abstracts

\begin{tabular}{llll}
\hline & Published & & $p$ value \\
\cline { 2 - 3 } & No $[n=383]$ & Yes $[n=158]$ & \\
\hline Session & & & $<0.001$ \\
Oral presentation & $7(1.8)$ & $13(8.2)$ & \\
Quick shot & $5(1.3)$ & $13(8.2)$ & \\
Poster & $371(96.9)$ & $132(83.5)$ & 0.34 \\
Type of data & & & \\
Clinical trial & $18(4.7)$ & $11(7.0)$ & \\
Retrospective & $354(92.4)$ & $140(88.6)$ & \\
Basic science & $11(2.9)$ & $7(4.4)$ & \\
Institution & & & \\
USA & $294(76.8)$ & $124(78.5)$ & \\
International & $82(21.4)$ & $28(17.7)$ & \\
Multinational & $7(1.8)$ & $6(3.8)$ & \\
Single institution & & & \\
No & $121(31.7)$ & $56(35.4)$ & \\
Yes & $262(68.3)$ & $102(64.6)$ & \\
\hline
\end{tabular}

Data are expressed as $n(\%)$ 
published as full manuscripts were published in ASO. Oral presentations (92.3\%) and quick shot presentations (84.6\%) were more likely to be published in ASO, while international studies were less likely to be published in ASO (Table 2). There was no significant association between type of study and publication likelihood in ASO. Abstracts published in journals other than ASO had a significantly greater lag time to publication (6.479 months \pm 6.487 vs. 14.049 months $\pm 8.409 ; p<0.001)$. Abstracts not published in ASO were published in journals with a significantly lower impact factor $(4.16$ vs. 3.63; $p=0.039$ ); however, there was no correlation with time to publication and impact factor (Fig. 1). Oral presentations were more likely to be published in a higher impact journal, while type of data or institution characteristics had no association with publication in a journal with a higher impact factor (Table 3). The journals in which other manuscripts were published can be found in electronic supplementary Table 1 .

Next, as we found $29.2 \%$ of the abstracts presented at the 2017 and 2018 ASBrS conferences led to full publication in a peer-reviewed journal, we sought to compare this with breast abstracts presented at the 2018 SSO conference. The 2018 SSO meeting resulted in 54 of 111 (48.6\%) breast abstracts leading to full publication, resulting in a significantly higher publication rate in any journal (Table 4); however, there was no difference in the percentage of publications in ASO, the mean impact factor of journals where the studies were published, or lag time to publication. Again, there was no correlation between impact factor and time to publication (Fig. 1). More

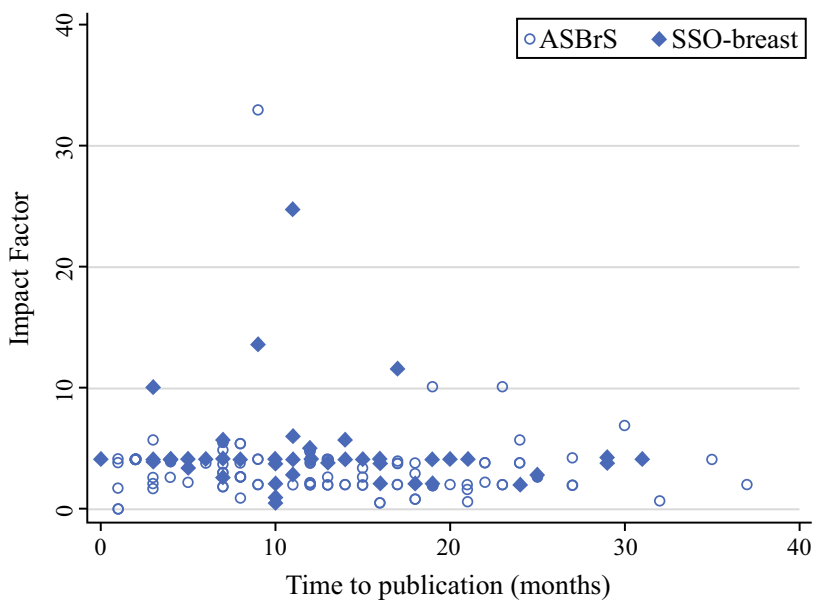

FIG. 1 Association of impact factor and time to publication $(r=-0.0856, p=0.220)$. ASBrS American Society of Breast Surgeons, SSO Society of Surgical Oncology

clinical trial data and basic science research were presented at the SSO meeting compared with the ASBrS meeting, while more studies from the ASBrS meeting contained retrospective data. The ASBrS meeting had a greater percentage of international studies and abstracts presented as posters (Table 4).

\section{DISCUSSION}

The scientific rigor of the abstracts presented at the $\mathrm{ASBrS}$ meeting, and breast abstracts presented at the SSO meeting, has not been recently evaluated. In this study, we demonstrated that in total, $29.2 \%$ of abstracts presented at
TABLE 2 Factors associated with publication of American Society of Breast Surgeons Conference abstracts in Annals of Surgical Oncology

\begin{tabular}{llll}
\hline & \multicolumn{2}{l}{ Published in Annals of Surgical Oncology } & $p$ value \\
\cline { 2 - 3 } & No $[n=83]$ & Yes $[n=75]$ & \\
\hline Session & $1(7.96)$ & $12(92.31)$ & \\
Oral presentation & $2(15.38)$ & $11(84.62)$ & 0.001 \\
Quick shot & $80(60.61)$ & $52(39.39)$ & \\
Poster & & $6(54.55)$ & 0.016 \\
Type of data & $5(45.45)$ & $65(46.43)$ & \\
Clinical trial & $75(53.57)$ & $4(57.14)$ & \\
Retrospective & $3(42.86)$ & $66(53.23)$ & \\
Basic science & & $7(25.00)$ & 0.506 \\
Institution & $58(46.77)$ & $2(33.33)$ & \\
USA & $21(75.00)$ & $29(51.79)$ & \\
International & $4(66.67)$ & $46(45.10)$ & \\
Multinational & $27(48.21)$ & $56(54.90)$ &
\end{tabular}

Data are expressed as $n(\%)$ 
TABLE 3 Impact factor stratified by session type for American Society of Breast Surgeons Conference abstracts

\begin{tabular}{llll}
\hline & Standard error & $p$ value & 95\% CI \\
\hline Session & & & \\
Quick shot versus poster & 0.7569953 & 0.806 & -1.317909 to 2.264867 \\
$\quad$ Oral presentation versus poster & 0.7569953 & 0.001 & 1.120553 to 4.703328 \\
$\quad$ Oral presentation versus quick shot & 1.021436 & 0.047 & 0.0212894 to 4.855634 \\
Type of data & & & \\
Retrospective versus clinical trial & 0.8532438 & 0.997 & -2.079499 to 1.95881 \\
Basic science versus clinical trial & 1.317455 & 0.960 & -2.759244 to 3.476127 \\
Basic science versus retrospective & 1.055336 & 0.917 & -2.078608 to 2.91618 \\
Institution & & & -2.206156 to 3.570442 \\
Multinational versus international & 1.220522 & 0.842 & -0.6477396 to 2.038961 \\
US versus international & 0.5676659 & 0.440 & -2.670285 to 2.697221 \\
US versus multinational & 1.134086 & 1.000 & \\
\hline
\end{tabular}

CI confidence interval

\begin{tabular}{|c|c|c|c|}
\hline & $\begin{array}{l}\mathrm{ASBrS} \\
{[n=541]}\end{array}$ & $\begin{array}{l}\text { SSO } \\
{[n=111]}\end{array}$ & $p$ value \\
\hline Session $[n(\%)]$ & & & $<0.001$ \\
\hline Oral presentation & $20(3.7)$ & 24 (21.6) & \\
\hline Quick shot & $18(3.3)$ & $10(9.0)$ & \\
\hline Poster & $503(93.0)$ & $77(69.4)$ & \\
\hline Published $[n(\%)]$ & & & $<0.001$ \\
\hline Yes & $158(29.2)$ & $54(48.6)$ & \\
\hline No & $383(70.8)$ & $57(51.4)$ & \\
\hline Annals of Surgical Oncology $[n(\%)]$ & & & 0.88 \\
\hline Yes & $75(47.8)$ & $26(49.1)$ & \\
\hline No & $83(52.2)$ & $28(50.9)$ & \\
\hline Impact factor & $3.6896835(2.7088485)$ & $4.5596154(3.6088812)$ & 0.067 \\
\hline Type of data $[n(\%)]$ & & & $<0.001$ \\
\hline Clinical trial & $29(5.4)$ & $12(10.8)$ & \\
\hline Retrospective & $494(91.3)$ & $79(71.2)$ & \\
\hline Basic science & $18(3.3)$ & $20(18.0)$ & \\
\hline Institution $[n(\%)]$ & & & 0.004 \\
\hline International & $110(20.3)$ & $10(9.4)$ & \\
\hline Multinational & $13(2.4)$ & $6(5.4)$ & \\
\hline USA & $418(77.3)$ & $95(85.6)$ & \\
\hline Single institution $[n(\%)]$ & & & 0.19 \\
\hline Yes & $363(67.2)$ & 67 (39.6) & \\
\hline No & $178(32.8)$ & $44(39.6)$ & \\
\hline Lag time to publication & $10.483871(8.4402828)$ & 11.685185 (7.503016) & 0.36 \\
\hline
\end{tabular}

TABLE 4 American Society of Breast Surgeons Conference versus Society of Surgical Oncology Conference publication comparison

$A S B r S$ American Society of Breast Surgeons, SSO Society of Surgical Oncology

the ASBrS 2017 and 2018 meetings led to full publication in a peer-reviewed journal. In 2017, the ASBrS publication rate was $21.6 \%$, while in 2018 , the publication rate was found to be $36.6 \%$. Of the 111 breast abstracts presented at the 2018 SSO meeting, 54 (48.6\%) were published as a full manuscript, resulting in a significant difference in the publication rate between the two conferences. However, there was no significant difference in the rate of publication to ASO between the abstracts presented at the two meetings. 
It is unknown as to the reason for the increase in the publication rate between the 2017 and 2018 ASBrS conferences. One may expect proceeding conferences to have a higher publication rate as it allows a greater period of time to complete a project. The publication rate for the ASBrS 2019 conference was, expectedly, found to be lower than the 2018 meeting, at $28.3 \%$ (electronic supplementary Table 1), and the 2019 SSO conference was lower, at $38.9 \%$ (electronic supplementary Table 2). We speculate the difference noted in the publication rate between the ASBrS conference and the SSO conference could be related to the bias in which abstracts are sent to each conference. Although the ASBrS and SSO conferences both focus on breast disease, allowing for the comparison of publication rates, the ASBrS conference focuses solely on breast disease, while the SSO conference focuses on multiple disease sites. The conferences could attract different subspecialties or trainees, which could potentially influence possible publication rates. Additionally, we were unable to discern if the primary author of the study was a trainee or attending physician, which may also influence publication rates.

Regardless of the conference, there was a higher publication rate for oral presentations in higher impact journals, indicating that the abstract review process properly stratifies research presented at these meetings. The higher impact trend for oral presentations was also noted in a previous study evaluating the publication rate of abstracts presented at the annual meetings of the Society of University Surgeons (SUS) and the Association for Academic Surgery (AAS). ${ }^{9}$ Additionally, a recent Cochrane review found that $37.3 \%$ of abstracts were published as a full manuscript. Studies that had positive results demonstrated an association with full publication, as did oral presentations, randomized trials, basic research, and abstracts originating from academic institutions. ${ }^{8}$ The results from that review are on a par with our findings. In comparison, Housri et al. investigated the publication rate from 1200 abstracts presented at the SUS and the AAS, and found that the SUS had a publication rate of $68.9 \%$, while the AAS had a publication rate of $53 \% .^{9}$

Surgical conferences have evolved over the years. Scientific meetings provide opportunities beyond knowledge sharing, including reuniting with former colleagues as well as providing opportunities for networking for prospective employers and research collaborators. Conferences in multiple specialties have been seen as providing the most up-to-date information to practitioners in order to disseminate the information. ${ }^{1}$ However, in the age of advancing technology and the ability to obtain information almost instantly has called into question the utility of obtaining new information at these conferences. Additionally, the scientific rigor at which data presented at conferences are reviewed is at times called into question. Studies have shown data can change substantially between abstract presentation and final publication, highlighting the importance of full peer review. ${ }^{4,5}$ Therefore, we believe our reported publication rate of $29.2 \%$ for abstracts presented at the ASBrS 2017 meeting and $48.6 \%$ for the 2018 SSO meeting are excepted as some studies may fail the rigors of full peer review. Furthermore, bias does exist in the medical literature. Publication bias may occur when the publication of research depends on whether the study produced positive or negative results, and may in fact be a barrier to submission for full publication. ${ }^{8,10,11}$ Similarly, upon questioning authors who failed to publish their abstract, common reasons for not publishing included that there was not enough time to prepare a manuscript, studies were still ongoing, or the responsibility of writing the manuscript belonged to someone else. ${ }^{12}$

Social media has also changed the landscape on how information is disseminated and made available to both medical professionals and non-physicians. The use of platforms such as Twitter have become an important method for the transfer of information. Interestingly, the use of Twitter in conjunction with surgical meetings has recently been evaluated. The use of Twitter at the ASBrS annual meeting has increased 469\% from 2013 to 2016, becoming a real-time communication tool. ${ }^{13}$ However, this number may even be an underrepresentation, as an additional study demonstrated only about $50 \%$ of tweets contained the 'hashtag' for the European SSO 39th clinical conference. ${ }^{14}$

The ongoing COVID-19 pandemic has greatly changed many aspects of how we conduct our daily lives. In addition, the COVID-19 pandemic has led to many conferences being postponed and/or held in a virtual format. ${ }^{15}$ For example, the 2020 SSO conference was postponed and was held as a virtual format, with plans for the 2021 conference to also be held virtually. Data have shown that attendance at grand rounds has significantly increased using the virtual format, and may in fact decrease the stress of having to take the time to attend in person. ${ }^{16,17}$ Similarly, as conferences are now being held virtually, this called into question how the virtual format will impact attendance and the overall number of abstracts submitted, as one can now present without the hassle of travel. Some argue the use of the virtual format will make conferences more affordable, less time-consuming, and more accessible worldwide. ${ }^{18} \mathrm{~A}$ recent study found that $188 / 273(69 \%)$ registered participants attended the live virtual Trans Tasman Radiation Oncology Group (TROG) conference, with a majority indicating they would be interested in a virtual meeting in the future. ${ }^{19}$ Additionally, groups have laid out best practice guidelines to help streamline the virtual process. ${ }^{20}$ 
As conferences have evolved into a virtual format, we hope the same review process would occur to keep scientific standards high, as virtual meetings offer the ability to quickly and widely disseminate information without sufficient vetting by moderators during sessions to ensure sound scientific basis. ${ }^{21}$ Our study evaluating the publication rate of ASBrS and SSO breast topic abstracts before the COVID-19 pandemic, and before widespread use of virtual conferences, offers a baseline metric for which the new virtual format can be assessed. Additionally, the virtual format no longer allows for the same networking opportunities, as in-person events and some industries, such as drug, device, and biotechnology, may suffer from lack of physician interaction. ${ }^{1}$ However, use of the virtual format will aid to solve some issues, such as the inability to accommodate all those who wish to participate in a particular session, as well as the cost, in order to organize and set-up for the meeting. ${ }^{22}$

\section{CONCLUSION}

Our study demonstrated a publication rate of $29.2 \%$ for abstracts presented at the 2017 and 2018 ASBrS conference and $48.6 \%$ from breast abstracts presented at the 2018 SSO conference. The question of how conferences will proceed in the future after the COVID-19 pandemic is still in flux, with modern technology and social media providing the opportunity to interact with people from all over the world. Our study provides a baseline metric for comparing the scientific rigor of future conferences, either virtual or inperson. Future studies are required on how the virtual format will affect data presented at scientific meetings.

\section{ACKNOWLEDGMENTS None.}

AUTHOR'S CONTRIBUTIONS ZB and KUP conceived and designed the project; $\mathrm{ZB}$ and $\mathrm{AL}$ obtained and organized the data; and CS performed the statistical analysis. All authors contributed to writing and revising this manuscript.

FUNDING Not applicable.

DISCLOSURES Zachary J. Brown, Amy Li, Chengli Shen, and Ko Un Park declare they have no competing interests.

\section{REFERENCES}

1. Ioannidis JP. Are medical conferences useful? And for whom? JAMA. 2012;307(12):1257-8.

2. Murrey DB, Wright RW, Seiler JG 3rd, Day TE, Schwartz HS. Publication rates of abstracts presented at the 1993 annual Academy meeting. Clin Orthop Relat Res. 1999;359:247-53.

3. Ross JS, Gross CP, Desai MM, et al. Effect of blinded peer review on abstract acceptance. JAMA. 2006;295(14):1675-80.
4. Toma M, McAlister FA, Bialy L, Adams D, Vandermeer B, Armstrong PW. Transition from meeting abstract to full-length journal article for randomized controlled trials. JAMA. 2006;295(11):1281-7.

5. Weintraub WH. Are published manuscripts representative of the surgical meeting abstracts? An objective appraisal. J Pediatr Surg. 1987;22(1):11-3.

6. Stephenson CR, Vaa BE, Wang AT, et al. Conference presentation to publication: a retrospective study evaluating quality of abstracts and journal articles in medical education research. BMC Med Educ. 2017;17(1):193.

7. Cook DJ, Guyatt GH, Ryan G, et al. Should unpublished data be included in meta-analyses? Current convictions and controversies. JAMA. 1993;269(21):2749-53.

8. Scherer RW, Meerpohl JJ, Pfeifer N, Schmucker C, Schwarzer G, von Elm E. Full publication of results initially presented in abstracts. Cochrane Database Syst Rev. 2018;11(11):Mr000005.

9. Housri N, Cheung MC, Gutierrez JC, Zimmers TA, Koniaris LG. SUS/AAS abstracts: what is the scientific impact? Surgery. 2008;144(2):322-31.

10. Kien C, Nußbaumer B, Thaler KJ, et al. Barriers to and facilitators of interventions to counter publication bias: thematic analysis of scholarly articles and stakeholder interviews. BMC Health Serv Res. 2014;14:551.

11. Carroll HA, Toumpakari Z, Johnson L, Betts JA. The perceived feasibility of methods to reduce publication bias. PloS ONE. 2017;12(10):e0186472.

12. Sprague S, Bhandari M, Devereaux PJ, et al. Barriers to full-text publication following presentation of abstracts at annual orthopaedic meetings. J Bone Jt Surg Am. 2003;85(1):158-63.

13. Attai DJ, Radford DM, Cowher MS. Tweeting the meeting: Twitter use at The American Society of Breast Surgeons Annual Meeting 2013-2016. Ann Surg Oncol. 2016;23(10):3418-22.

14. Mackenzie G, Søreide K, Polom K, et al. Beyond the hashtag-an exploration of tweeting and replies at the European Society of Surgical Oncology 39th clinical conference (ESSO39). Eur J Surg Oncol. 2020;46(7):1377-83.

15. Vervoort D, Ma X, Bookholane H, Nguyen TC. Conference cancelled: the equitable flip side of the academic surgery coin. Am J Surg. 2020;220(6):1539-40.

16. Lebares CC, Guvva EV, Desai A, et al. Key factors for implementing mindfulness-based burnout interventions in surgery. Am J Surg. 2020;219(2):328-34.

17. Sutzko DC, Martin CA, Chu DI. Development and implementation of virtual grand rounds in surgery. Am J Surg. 2021;221(1):46-8.

18. Sarabipour S. Virtual conferences raise standards for accessibility and interactions. Elife. 2020;9:e62668.

19. McDowell L, Goode S, Sundaresan P. Adapting to a global pandemic through live virtual delivery of a cancer collaborative trial group conference: the TROG 2020 experience. J Med Imaging Radiat Oncol. 2020;64(3):414-21.

20. Rubinger L, Gazendam A, Ekhtiari S, et al. Maximizing virtual meetings and conferences: a review of best practices. Int Orthop. 2020;44(8):1461-6.

21. Gupta MP, Sridhar J, Wykoff CC, Yonekawa Y. Ophthalmology conferences in the coronavirus disease 2019 era. Curr Opin Ophthalmol. 2020;31(5):396-402.

22. Lecueder S, Manyari DE. Virtual congresses. J Am Med Inform Assoc. 2000;7(1):21-7.

Publisher's Note Springer Nature remains neutral with regard to jurisdictional claims in published maps and institutional affiliations. 